\title{
Supplementation of a high-fat diet with acesulfame-k results in sex-specific effects on insulin concentrations and adipose tissue morphology and gene expression in C57BL6 mice
}

\author{
P.E. Bridge-Comer ${ }^{1}$, M.H. Vickers ${ }^{1}$ and C.M. Reynolds ${ }^{1,2}$ \\ ${ }^{1}$ The Liggins Institute, University of Auckland, Auckland, New Zealand and \\ ${ }^{2}$ School of Public Health, Physiotherapy and Sports Science, Conway Institute, Institute of Food and Health, UCD, \\ Belfield, Dublin, Ireland
}

High fat diet (HFD) and sugar consumption are associated with obesity and metabolic disease. Artificial sweeteners (AS) are often used as replacements in diet or 'lite' foods/beverages. Despite being one of the most prevalent sweeteners consumed in Ireland, acesulfame-K (Ace-k) is relatively understudied ${ }^{(1)}$. There are conflicting reports around the efficacy of substituting sugar with AS in relation to metabolic disease risk $^{(2)}$, and evidence for the safety of AS in combination with HFD are lacking. We examined the effects of HFD and Ace-K consumption on glucose tolerance and adipose tissue physiology in male and female mice.

$40 \mathrm{C} 57 \mathrm{Bl} / 6$ mice (20 male and 20 female - 10 weeks old) were randomized to receive a) Control diet (CDCon; standard chow diet/ water), b) Ace-k (AS) control diet (CDAS; standard chow diet/7.5 mM AS in drinking water), c) HFD (HFCon; HFD (45\%kcal from fat) /water), d) HF and AS diet (HFAS; HFD (45\%kcal from fat) $/ 7.5 \mathrm{mM} \mathrm{AS}$ in drinking water) for 6 weeks. $\mathrm{N}=5 \mathrm{males}$ and $\mathrm{n}=5$ females per group. Body weight and food intake were assessed weekly. An oral glucose tolerance test (OGTT) was carried out after 5 weeks on the experimental diet and animals were killed after 6 weeks. Insulin and leptin were determined by ELISA. Adipose tissue morphology was assessed by haemotoxylin and eosin staining. Data was analysed by 2-way ANOVA with HF diet and AS supplementation as factors.

HFD increased body weight in male and female mice irrespective of AS supplementation $(\mathrm{P}=0.02, \mathrm{P}=0.002$, respectively). HFD consumption significantly impaired glucose tolerance in male and female mice $(\mathrm{P}<0.0001, \mathrm{P}=0.03$, respectively). AS supplementation improved glucose tolerance in CDAS $(\mathrm{P}=0.04)$ and HFAS $(\mathrm{P}=0.045)$ groups in male but not female mice. This was associated with a reduction in plasma insulin concentrations in the HFAS group $(P=0.03)$. In female mice, there was a significant increase in plasma insulin concentrations in HFAS compared to HFCon groups $(\mathrm{P}=0.01)$. HFD consumption increased gonadal adipose tissue weight in males and females $(\mathrm{P}=0.005, \mathrm{P}=0.006$, respectively), this was associated with an increase in adipocyte size and plasma leptin concentrations $(\mathrm{P}<0.05)$. While there was an increase in HFAS gonadal fat weight in males, average adipocyte size was decreased $(\mathrm{P}=0.04)$. In contrast, female CDAS and HFAS had decreased gonadal fat weight but no relative change in average adipocyte size or leptin concentration.

HFD has a negative effect on adiposity, glucose tolerance and insulin secretion in male and female mice. Supplementation of HFD with AS has significant sex-specific effects, with positive alterations in glucose tolerance and insulin secretion accompanied by reduced adipocyte size in males and increased glucose intolerance and insulin secretion in females. This highlights the need for inclusion of both sexes in basic research and subsequent development of tailored public health messages.

\section{References}

1. Buffini M, Goscinny S, Loco JV, et al. (2018) Food Additives \& Contaminants: Part A 35(3), 425-38.

2. Young J, Conway EM, Rother KI, et al. (2019) Pediatr Obes 14(8) e12521. 